\title{
The latest X-ray analysis solutions from Anton Paar
}

\author{
A. O. F. Jones, M. Kremer, T. Müller, B. Puhr, B. Schrode \\ Anton Paar GmbH, Graz, Austria \\ andrew.jones@anton-paar.com
}

$\mathrm{X}$-ray based analysis techniques play a crucial role in a vast range of academic and industrial research areas. These include fields as diverse as pharmaceutical research, geology, building materials, materials science, specialty chemicals, and the life sciences. X-ray based methods can be advantageous over complementary methods such as electron microscopy due to the minimum need for sample preparation, the non-destructive nature of X-rays, and the possibility to work under both ambient and non-ambient conditions (in-situ studies). In addition, different techniques such as XRD and SAXS may both be used together to give complementary information which allows a more in-depth understanding of the sample in question and its properties.

As a manufacturer of X-ray sources, advanced X-ray optics, XRD equipment, and SAXS instruments, this poster will present the latest developments in X-ray analysis equipment from Anton Paar which further extend the capabilities of in-house X-ray based measurements under both ambient and non-ambient conditions.

Keywords: Powder XRD; SAXS; non-ambient XRD; in-situ studies 